\title{
Frontal Eye Field Neurons Orthodromically Activated From the Superior Colliculus
}

\author{
MARC A. SOMMER AND ROBERT H. WURTZ \\ Laboratory of Sensorimotor Research, National Eye Institute, National Institutes of Health, Bethesda, \\ Maryland 20892-4435
}

\begin{abstract}
Sommer, Marc A. and Robert H. Wurtz. Frontal eye field neurons orthodromically activated from the superior colliculus. J. Neurophysiol. 80: 3331-3333, 1998. Anatomical studies have shown that the frontal eye field (FEF) and superior colliculus (SC) of monkeys are reciprocally connected, and a physiological study described the signals sent from the FEF to the SC. Nothing is known, however, about the signals sent from the SC to the FEF. We physiologically identified and characterized FEF neurons that are likely to receive input from the SC. Fifty-two FEF neurons were found that were orthodromically activated by electrical stimulation of the intermediate or deeper layers of the SC. All the neurons that we tested $(n=34)$ discharged in response to visual stimulation. One-half also discharged when saccadic eye movements were made. This provides the first direct evidence that the ascending pathway from SC to FEF might carry visual- and saccade-related signals. Our findings support a hypothesis that the SC and the FEF interact bidirectionally during the events leading up to saccade generation.
\end{abstract}

\section{INTRODUCTION}

The frontal eye field (FEF) and the superior colliculus (SC) are both involved in the generation of rapid, or saccadic, eye movements. Reversible inactivation of either structure impairs saccades (e.g., Sommer and Tehovnik 1997; Hikosaka and Wurtz 1985), and combined ablation of both structures results in permanent saccadic deficits (Schiller et al. 1980). Anatomic studies have shown that the FEF and the SC are reciprocally connected (for review see Schall 1997). A previous physiological study examined the FEF projection to SC (Segraves and Goldberg 1987), but functional characteristics of the ascending pathway, from SC to FEF (presumably via thalamus) (Lynch et al. 1994), have never been described. The purpose of this study was to identify and characterize FEF neurons that can be activated from the SC via synapses. A description of these neurons would provide crucial information for evaluating whether the ascending pathway plays a role in the generation of saccades.

\section{METHODS}

Chambers for accessing the FEF and SC were implanted on two monkeys (Macaca mulatta) using surgical techniques described previously (Munoz and Wurtz 1993). All procedures were ap-

The costs of publication of this article were defrayed in part by the payment of page charges. The article must therefore be hereby marked "advertisement" in accordance with 18 U.S.C. Section 1734 solely to indicate this fact. proved by the Institute Animal Care and Use Committee and complied with Public Health Service Policy on the humane care and use of laboratory animals. The FEF and SC were located anatomically with magnetic resonance imaging and physiologically by recording from saccade-related neurons and by evoking saccades at $<50-\mu \mathrm{A}$ threshold. Two tungsten microelectrodes for stimulation were implanted in the intermediate layers of the ipsilateral SC (typically 1 was placed at $<3^{\circ}$ eccentricity and 1 between 10 and $30^{\circ}$ eccentricity on the SC map, Robinson 1972) or one was moved through the SC in a penetration (at $8^{\circ}$ eccentricity). We recorded from single FEF neurons with conventional methods and attempted to activate each with SC stimulation (single biphasic pulse, 0.15 $\mathrm{ms} /$ phase, $\leq 600 \mu \mathrm{A} /$ phase; Fig. $1 A$ ).

An FEF neuron was considered activated if it discharged consistently ( $\geq 1 / 3$ of stimulation trials) at about the same latency (variability $<2 \mathrm{~ms}$ ) after stimulation. We performed the collision test on each activated neuron, and those that passed were identified as antidromically activated (for review see Lemon 1984). Failure of the collision test, a negative result, suggests orthodromic activation but requires confirmation. If the neurons failing the collision test were truly activated via synapses, then as a group their relative refractory periods, latencies, and latency variances should be larger than those of the antidromically activated neurons. We measured relative refractory periods with a method based on that of Fuller and Schlag (1976). A pair of 0.05-ms, monophasic cathodal pulses was delivered to the SC at 1.2 times current threshold, and the temporal interval between the pulses was decreased until the FEF neuron was activated by the second pulse on only $50 \%$ of trials; this interval was the relative refractory period. We measured latencies of activation and range of latency variance (the " jitter") by using the technique of Swadlow et al. (1978) in which we examined the response to the second of a pair of SC stimulation pulses (each biphasic, $0.15 \mathrm{~ms} /$ phase) delivered at two to three times threshold and separated by $4 \mathrm{~ms}$ (or longer if the relative refractory period was $\geq 4 \mathrm{~ms}$ ).

We had the monkeys perform two oculomotor tasks that permitted general characterization of the neurons' visual- and saccaderelated properties. Visual targets, $0.3 \times 0.3^{\circ}$, were back-projected onto a tangent screen with a liquid crystal display (LCD) projector and placed at the optimal location for eliciting neural responses as estimated during preliminary testing. Eye position was measured with implanted scleral search coils. In all trials, the monkey first fixated a spot of light in the center of the screen. A visual target then appeared and remained lit in half of the trials (visual task) or disappeared after $100 \mathrm{~ms}$ in the other half (memory task). A delay period of $500-1,000 \mathrm{~ms}$ ensued. After this period, the fixation spot disappeared, cueing the monkey to make a saccade to the visible or remembered target location. The visual and memory trials were pseudorandomly interleaved. Four epochs of firing rate were defined for each trial: baseline, 500-300 ms before target onset (during fixation); visual, 50-200 ms after target onset; delay, 300-0 ms before the cue to move; and saccade, 100-0 ms before 


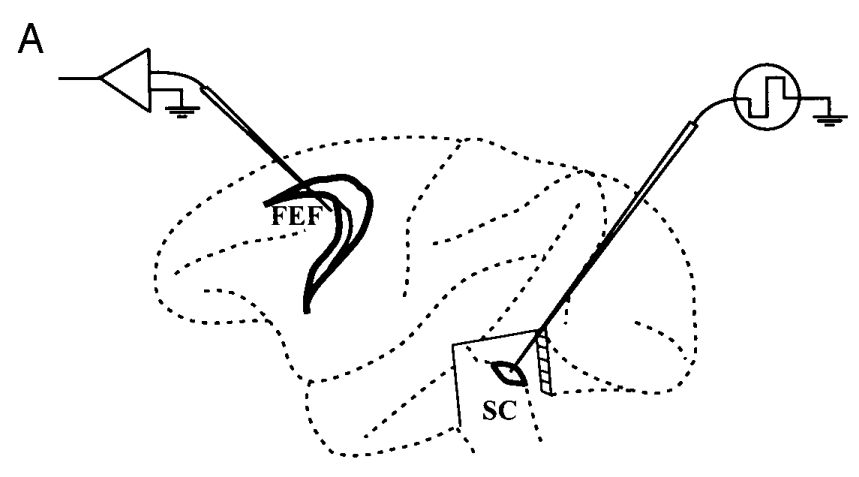

B
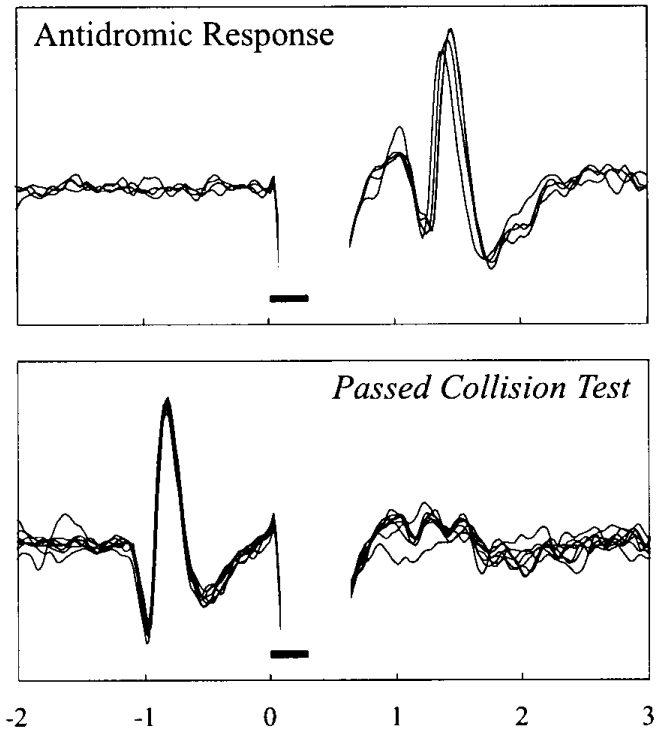

C
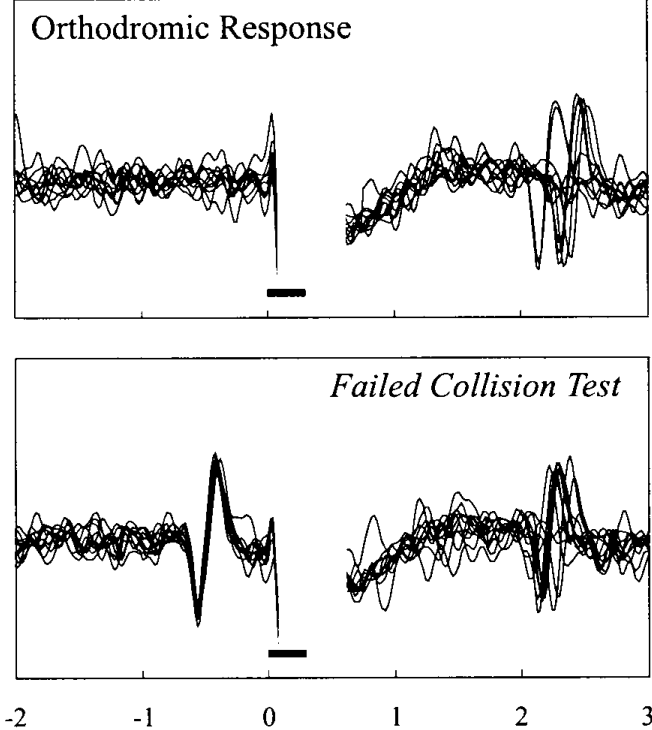

Time Relative to SC stimulation (ms)
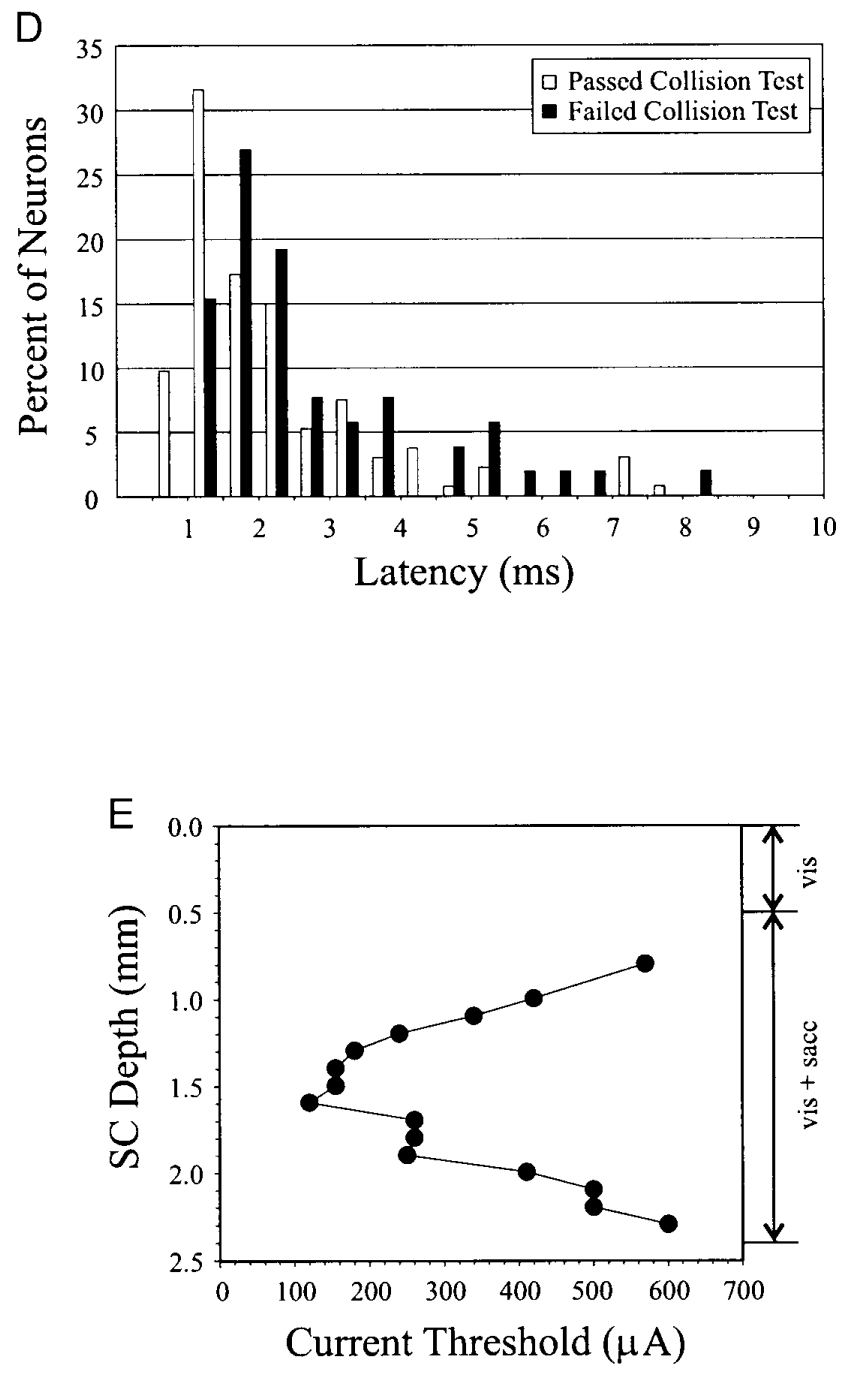
saccade initiation. We subjected all 8 groups ( 4 epochs $\times 2$ tasks ) to a one-way analysis of variance, and if this was significant $(P<$ 0.01 ) we ran an all-pairwise multiple comparison test (StudentNewman Keuls or Dunn's, $P<0.05)$. A neuron had visual or delay activity if, in either task, the firing rate in the respective epoch significantly exceeded the baseline firing rate. A neuron had saccadic activity if, in either task, the saccade firing rate significantly exceeded the delay firing rate. We considered the delay or saccadic activity to be influenced by the presence of a visual target if the firing rate in the respective epoch was significantly greater in visual than in memory trials.

\section{RES ULTS}

We found 185 FEF neurons activated by SC stimulation. The collision test was performed on each neuron by using the neuron's spontaneous discharge to trigger stimulation. A neuron passed the collision test and therefore was antidromically activated if there was a time period after its spontaneous discharge during which SC stimulation was unable to cause activation. This prevention of activation occurs for antidromically activated neurons because the stimulationevoked action potential traveling toward the soma collides with the spontaneously evoked action potential traveling away from the soma (neither action potential propagates further because the axon is in its absolute refractory period on both sides of the collision point). One hundred thirtythree FEF neurons passed the collision test and therefore were known to be antidromically activated. For example, responses of a neuron activated at 1.0-ms latency are shown in Fig. $1 B$. Activation of the neuron was prevented if SC stimulation occurred $\leq 1.4 \mathrm{~ms}$ after the neuron discharged spontaneously (Fig. $1 \mathrm{~B}$, bottom). In contrast, 52 neurons failed the collision test. The neuron shown in Fig. $1 C$ was activated from the SC after a latency that averaged $\sim 2.2 \mathrm{~ms}$ with 0.3-ms jitter (Fig. 1C, top ), and activation still occurred if SC stimulation was delivered immediately after the neuron discharged (Fig. $1 C$, bottom). We found no interval between the spontaneous discharge and stimulation that prevented activation (intervals from 0.5 to $10 \mathrm{~ms}$ were tried, a range that should have been more than adequate) (see Fuller and Schlag 1976 for details regarding the collision interval). The 52 FEF neurons that failed the collision test probably were activated from the SC orthodromically, via synapses.

Further evidence supported our interpretation that the 52 FEF neurons failing the collision test were orthodromically activated. Activation latencies of neurons failing the collision test (Fig. 1D; minimum $1.2 \mathrm{~ms}$; mean $2.7 \pm 1.6 \mathrm{~ms}$; mean $\pm \mathrm{SD}$; median $2.1 \mathrm{~ms} ; n=52$ ) were longer than those of the known antidromically driven neurons passing the collision test (minimum $0.7 \mathrm{~ms}$; mean $2.1 \pm 1.5$; median 1.6 ms; $n=133$; Mann-Whitney rank sum test, $P<0.001)$.
Latency jitters for neurons that failed the collision test (mean $0.5 \pm 0.5 \mathrm{~ms} ; n=26$ ) were longer than for neurons that passed ( $<0.1 \mathrm{~ms}$ for $97 \%$ of the neurons; $n=75$; note, we could not accurately quantify jitters $<0.1 \mathrm{~ms}$ ). Finally, the relative refractory periods of neurons failing the collision test (mean $5.7 \pm 7.2 \mathrm{~ms} ; n=29$ ) were longer than those of neurons passing the collision test (mean $0.7 \pm 0.3 \mathrm{~ms}$; $n=79$; Mann-Whitney rank sum test, $P<0.001)$.

Current thresholds for orthodromically activating the 52 neurons ranged from 16 to $540 \mu \mathrm{A}$ (mean $115 \pm 97 \mu \mathrm{A}$ ). We estimate that these currents directly activated neural elements within $1.2 \mathrm{~mm}$ of the electrode tip (with the relationship Distance $=(\text { Current } / \mathrm{K})^{0.5}$ where $\mathrm{K} \cong 381 \mu \mathrm{A} / \mathrm{mm}^{2}$ for direct activation of low-threshold elements with $0.15-\mathrm{ms}$ duration cathodal pulses) (Tehovnik 1996). Because our stimulating electrodes were fixed in place with their tips in the SC intermediate layers (1.0-2.5 mm deep), all the FEF neurons probably were activated orthodromically by neural elements located within the SC. When we used moveable stimulating electrodes ( on 3 occasions), we found threshold minima in the intermediate and deeper SC layers (1.6-3.4 $\mathrm{mm}$ below the SC surface; e.g., Fig. $1 E$ ).

All of the orthodromically activated neurons that we tested in our visual and memory saccadic tasks had visual responses ( $n=34$; e.g., Fig. $2 A$, left). Visual receptive fields were peripheral (contralateral or on the vertical meridian) for $97 \%$ of neurons (33/34) and foveal for 3\% (1/34). Many neurons $(44 \%, 15 / 34)$ had delay activity, continuing to fire long after a target was presented (e.g., Fig. $2 \mathrm{~A}$, middle). For $73 \%$ of these neurons $(11 / 15)$, the delay activity was considered a tonic visual discharge because it required the presence of a visual target (i.e., the activity occurred in the visual but not the memory task). Saccadic activity was found in $50 \%$ (17/34) of the neurons (e.g., Fig. 2A, right). For 59\% of these neurons $(10 / 17)$, the saccadic activity required the presence of a visual target. In sum, all the neurons had visual responses, and when they had delay- and saccade-related discharges as well these often were enhanced by visual stimulation.

When we grouped our neurons according to their combinations of visual and saccadic discharges, as was done previously (e.g., Segraves and Goldberg 1987), we found that $50 \%$ of the neurons $(17 / 34)$ had only a visual response (e.g., Fig. $2 B$ ) and 50\% (17/34) had saccadic activity plus a visual response (e.g., Fig. 2A), but none of the neurons had only saccadic activity. We note that, in contrast, it was common to find pure saccadic discharges in nonactivated and antidromically activated neurons. We also note that there was no clear relation between an orthodromically activated neuron's discharge properties and the site on the SC map where we activated the neuron with least current.

FIG. 1. Experimental preparation and stimulation-related results. $A$ : we recorded from the frontal eye field (FEF) located in the rostral bank of the genu of the arcuate sulcus ( shown spread apart) while electrically stimulating the superior colliculus ( SC; shown in cutaway view). $B$ : success of the collision test. Bar above abscissa shows timing of SC stimulation (shock artifact is clipped, for clarity). Multiple trials are superimposed. Top panel: stimulation activated the neuron, eliciting an action potential after 1.0-ms latency. Bottom panel: a natural discharge of the neuron just before stimulation prevented activation of the neuron. $C$ : failure of the collision test. Stimulation elicited an action potential after $\sim 2.2$-ms latency, even after a natural discharge of the neuron. $D$ : histograms of activation latencies for neurons that passed or failed the collision test. $E$ : profile of current threshold vs. depth in the SC for 1 experiment that used a moveable stimulating electrode. Neural activity was recorded after finding the current threshold at each site: vis, visual activity only; vis + sacc, visual and saccadic activity. 


\section{IS C USS I ON}

We identified and characterized neurons in FEF that were driven from the SC via synapses. Possible routes of orthodromic activation and the implications of our findings will be discussed.

The only route of orthodromic activation from the SC to the FEF with a known anatomic basis is tectothalamocortical (Fig. 3A). A direct pathway from SC to mediodorsal thalamus to FEF was reported (Lynch et al. 1994). Furthermore, SC neurons that may contribute to this pathway were described anatomically; they seem to be located primarily in the superficial and intermediate layers, as suggested by the anatomic results of both Lynch et al. (1994), who labeled SC neurons with disynaptic retrograde transport of herpes virus from the FEF, and Moschovakis et al. (1988), who morphologically described SC neurons ( "L"' neurons) that project to the thalamus. Although we think the tectothalamocortical pathway is the most likely route of orthodromic
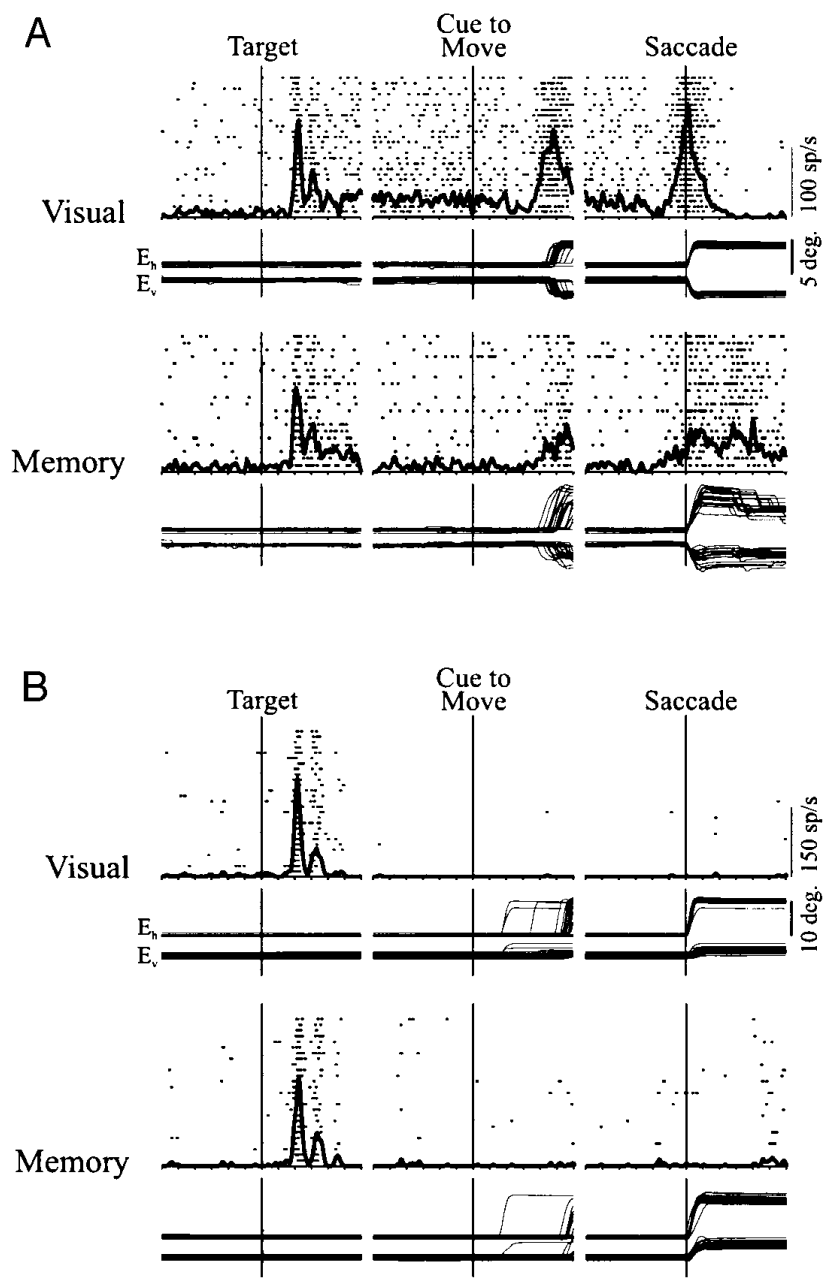

FIG. 2. Examples of discharge characteristics of FEF neurons orthodromically activated from the SC. A: activity of a neuron with visual-, delay-, and saccade-related discharges. For visual task (top row) and memory task (bottom row) trials, discharges in individual trials are shown in the rasters and are pooled in spike density functions (bold curves, Gaussian $\sigma=4.0$ $\mathrm{ms}$ ). Alignments are with target onset (left), the cue to move (middle), and saccadic initiation (right). Eye position shown as horizontal $\left(E_{\mathrm{h}}\right)$ and vertical $\left(E_{\mathrm{v}}\right)$ component traces. On the time axis (abscissa), each tic mark is separated by $50 \mathrm{~ms}$. $B$ : activity of a neuron with visual responses only.
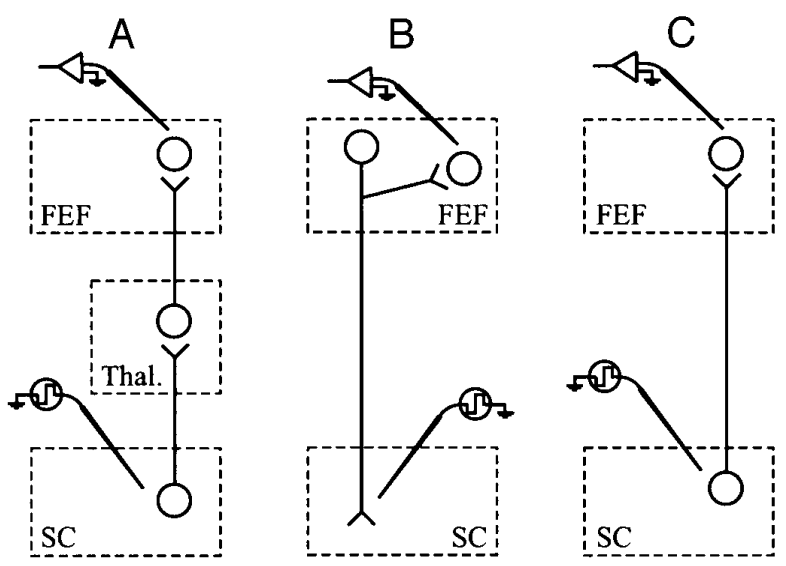

FIG. 3. Possible routes of orthodromic activation from the SC to the FEF. $\bigcirc$ : somata; lines depict projections. $A$ : known route proceeding disynaptically from SC to thalamus (Thal.) and then to FEF. $B$ : hypothetical route via a collateral that emerges from the axon of a corticotectal neuron. $C$ : hypothetical route via a direct monosynaptic projection from $\mathrm{SC}$ to FEF.

activation, two other routes also are hypothetically possible. First, stimulation-evoked action potentials might travel antidromically through the axon of a corticotectal neuron residing in FEF or elsewhere, invade collaterals, and then monosynaptically activate the recorded FEF neuron (Fig. $3 B$ ). It is not known, however, whether monkey FEF neurons do receive collaterals from corticotectal neurons. Also, it is questionable whether an antidromically evoked action potential could reliably invade a collateral because of the sudden increase in membrane area (and consequent drop in input resistance) that can occur at a branch point (reviewed by Swadlow et al. 1980). Finally, were an action potential to invade a collateral, it probably would travel toward the recorded FEF neuron at speeds too low to be consistent with our data. The conduction velocity in collaterals is $\sim 0.3 \mathrm{~m} /$ s (reviewed by Nowak and Bullier 1997) and therefore it can take $>3.0 \mathrm{~ms}$ for an action potential to propagate as little as $1,000 \mu \mathrm{m}$ through a collateral. The orthodromic activation latencies of our neurons seem too short to be attributable to such a slow route $($ Fig. $1 D)$. A third possible route of activation from $\mathrm{SC}$ to $\mathrm{FEF}$ is a direct monosynaptic one (Fig. $3 C$ ), but, to our knowledge, such a projection was never found in any of the numerous studies of FEF afferents (for review see Schall 1997).

The FEF neurons orthodromically activated from the SC are the first physiologically established candidates for receiving SC signals. As might be expected, many of the neurons exhibited saccade-related discharges. The significance of this putative relay of saccadic information from SC to FEF still needs to be determined. A more surprising finding was that every one of the neurons exhibited a visual response, suggesting that the SC might send visual-related activity to the FEF. If so, this relay of visual activity might play at least two roles. First, it might improve the overall signal-to-noise ratio of visual responses in the FEF. This would occur if the visual-related discharges sent to FEF from the SC are relatively stronger and less noisy than visual-related discharges sent from elsewhere (e.g., from extrastriate cortex). Second, visual signals sent from the SC might improve synchronization between it and the FEF. FEF visual responses 
driven by $\mathrm{SC}$ afferents might occur only a few milliseconds after SC visual responses because of the disynaptic pathway from $\mathrm{SC}$ to FEF.

Our results support the general hypothesis that the FEF and the SC interact with each other bidirectionally. It was known previously that there is a flow of visual- and saccaderelated signals from FEF to SC (Segraves and Goldberg 1987). Now we have direct evidence that the reciprocal pathway may carry visual- and saccade-related signals as well. Signals carried in both halves of the loop connecting the FEF and SC are likely to be important during the events leading up to saccade generation.

We thank the Laboratory of Diagnostic Radiology Research at the $\mathrm{Na}$ tional Institutes of Health for magnetic resonance images. We are grateful to Drs. Martin Paré and Serena Dudek for helpful discussions.

Address for reprint requests: M. A. Sommer, Laboratory of Sensorimotor Research, National Eye Institute, National Institutes of Health, Building 49, Room 2A50, 9000 Rockville Pike, Bethesda, MD 20892-4435.

Received 10 August 1998; accepted in final form 10 September 1998.

\section{REFERENCES}

Fuller, J. H. AND SChlag, J. D. Determination of antidromic excitation by the collision test: problems of interpretation. Brain Res. 112: $283-$ 298, 1976.

HikosaKa, O. AND WuRTZ, R. H. Modification of saccadic eye movements by GABA-related substances. I. Effect of muscimol and bicuculline in monkey superior colliculus. J. Neurophysiol. 53: 266-291, 1985.

LEMON, R. Methods for Neuronal Recording in Conscious Animals. IBRO
Handbook Series: Methods in the Neurosciences. New York: Wiley, 1984, vol. 4, p. 95-102.

LynCH, J. C., Hoover, J. E., AND STRICK, P. L. Input to the primate frontal eye field from the substantia nigra, superior colliculus, and dentate nucleus demonstrated by transneuronal transport. Exp. Brain Res. 100: 181186, 1994.

Moschovakis, A. K., Karabelas, A. B., and Highstein, S. M. Structurefunction relationships in the primate superior colliculus. I. Morphological classification of efferent neurons. J. Neurophysiol. 60: 232-262, 1988.

MunOz, D. P. AND WuRTZ, R. H. Fixation cells in monkey superior colliculus. I. Characteristics of cell discharge. J. Neurophysiol. 70: 559-575, 1993.

NowaK, L. G. AND Bullier, J. Cerebral Cortex. Extrastriate Cortex in Primates. New York: Plenum, 1997, vol. 12, p. 211-212.

Robinson, D. A. Eye movements evoked by collicular stimulation in the alert monkey. Vision Res. 12: 1795-1808, 1972.

Schall, J. D. Cerebral Cortex. Extrastriate Cortex in Primates. New York: Plenum, 1997, vol. 12, p. 589-594.

Schiller, P. H., True, S. D., AND Conway, J. L. Deficits in eye movements following frontal eye-field and superior colliculus ablations. J. Neurophysiol. 44: 1175-1189, 1980.

Segraves, M. A. AND Goldberg, M. E. Functional properties of corticotectal neurons in the monkey's frontal eye field. J. Neurophysiol. 58: $1387-$ 1419, 1987.

Sommer, M. A. And Tehovnik, E. J. Reversible inactivation of macaque frontal eye field. Exp. Brain Res. 116: 229-249, 1997.

Swadlow, H. A., Kocsis, J. D., AND Waxman, S. G. Modulation of impulse conduction along the axonal tree. Annu. Rev. Biophys. Bioeng. 9: $143-179,1980$

Swadlow, H. A., Waxman, S. G., And Rosene, D. L. Latency variability and the identification of antidromically activated neurons in mammalian brain. Exp. Brain Res. 32: 439-443, 1978.

TEHOVNIK, E. J. Electrical stimulation of neural tissue to evoke behavioral responses. J. Neurosci. Methods 65: 1-17, 1996. 